\title{
Trivium
}

Revue franco-allemande de sciences humaines et sociales - Deutsch-französische Zeitschrift für Geistesund Sozialwissenschaften

$31 \mid 2020$

La culture politique de la République romaine

\section{Jovialité et liberté}

À propos de la dimension institutionnelle des relations entre strates supérieures et inférieures dans la République romaine

\section{Martin Jehne}

Traducteur : Anthony Andurand

\section{(2) OpenEdition}

\section{Journals}

Édition électronique

URL : https://journals.openedition.org/trivium/7042

DOI : 10.4000 /trivium. 7042

ISBN : 1963-1820

ISSN : 1963-1820

\section{Éditeur}

Les éditions de la Maison des sciences de l'Homme

\section{Référence électronique}

Martin Jehne, "Jovialité et liberté », Trivium [En ligne], 31 | 2020, mis en ligne le 25 juin 2020, consulté le 23 août 2021. URL : http://journals.openedition.org/trivium/7042 ; DOI : https://doi.org/10.4000/ trivium. 7042

Ce document a été généré automatiquement le 23 août 2021.

\section{(c) (i) $\odot$}

Les contenus des la revue Trivium sont mis à disposition selon les termes de la Licence Creative Commons Attribution - Pas d'Utilisation Commerciale - Pas de Modification 4.0 International. 


\section{Jovialité et liberté}

À propos de la dimension institutionnelle des relations entre strates supérieures et inférieures dans la République romaine

\section{Martin Jehne}

Traduction : Anthony Andurand

\section{NOTE DE L'ÉDITEUR}

\section{Édition originale | Originalausgabe}

«Jovialität und Freiheit. Zur Institutionalität der Beziehungen zwischen Ober- und Unterschichten in der römischen Republik », in : Linke, B. / Stemmler, M. (éd.) : Mos Maiorum. Untersuchungen zu den Formen der Identititätstiftung und Stabilisierung in der römischen Republik, Stuttgart, Franz Steiner Verlag, 2000, p. 207-235.

Nous remercions M. Martin Jehne de nous avoir accordé l'autorisation de traduire ce texte pour le présent numéro.

Wir danken Herrn Martin Jehne für die freundliche Genehmigung, diesen Artikel in französischer Übersetzung zu publizieren.

Pour la traduction, un certain nombre de coupes a été réalisé dans le texte par l'auteur. Les passages supprimés sont indiqués par des crochets [...].

1 La question des fondements de la stabilité de la République romaine - restée dans l'ensemble si longtemps inébranlée, malgré de nombreux conflits - renvoie en fin de compte à celle de ses réalisations institutionnelles, dans la mesure où une fonction essentielle des institutions consiste à garantir de la continuité dans un processus de transformation ${ }^{1}$. Selon la définition opératoire proposée par le Sonderforschungsbereich «Institutionnalité et historicité" (Dresde), «le caractère institutionnel d'entités fondées sur la communication ou l'action apparaît dans des mécanismes ou des structures, qui, à travers la représentation symbolique que cette configuration véhicule d'elle-même, la stabilisent et la pérennisent, garantissent la probabilité, la répétitivité et la prédictibilité des faits sociaux. ${ }^{2}$ " Les réalisations institutionnelles de la République sont d'abord remarquables en ceci que l'ordre républicain tenait si 
intensément les citoyens sous son emprise que, même à la fin, il ne traversait que des crises, sans que des alternatives pussent être envisagées ${ }^{3}$. En outre, il est frappant que la res publica ait été en permanence dirigée par un petit groupe de familles sénatoriales ${ }^{4}$, bien que cette élite fût marquée en interne par un fort esprit de compétition $^{5}$ et ne possédât formellement, dans les affaires les plus importantes, aucun pouvoir de décision, lequel revenait davantage aux citoyens qui se rendaient dans les assemblées. Pour rendre compte de cette situation, il ne suffit pas de disséquer l'organisation formelle des assemblées et leurs règlements respectifs; il convient encore de s'intéresser aux formes standardisées à travers lesquelles les strates supérieures et les strates inférieures y communiquaient ${ }^{6}$. Il s'agit, en d'autres termes, d'analyser le caractère institutionnalisé des relations qui s'étaient établies entre les membres de la classe dirigeante et le peuple, incarné par les citoyens présents dans chaque assemblée. C'est à partir d'un exemple que j'aborderai plus précisément cette problématique.

$2 \mathrm{Au}$ livre IV de son Histoire romaine, Tite-Live évoque la terrifiante histoire du sévère tribun consulaire Postumius Regillensis, qui, après avoir injustement traité ses troupes lors de la répartition du butin et aggravé le conflit par une froide arrogance et de cruelles mesures disciplinaires, fut lapidé par ses soldats? ${ }^{7}$. C'est dans cet épisode transmis pour le compte de l'année 414 avant J.-C. que s'insère le récit d'un affrontement entre Postumius et le tribun de la plèbe M. Sextius lors d'une assemblée. Dans le cadre de sa lutte pour une loi agraire, Sextius avait annoncé qu'il demanderait également l'envoi de colons vers Bolae, puisqu'il était selon lui parfaitement légitime que ceux qui s'étaient emparés de Bolae par les armes possédassent la cité et ses environs ${ }^{8}$. Comme Bolae était la cité que Postumius venait de prendre avec ses troupes et qu'il n'avait, en dépit de ses promesses antérieures, pas laissée à ses soldats, l'argumentation de Sextius constituait une attaque évidente contre l'attitude de Postumius. Sur quoi le tribun consulaire, aisément irritable, se laissa aller à la formule suivante : « Malheur à mes soldats, s'ils remuent ! ${ }^{9}$ » Cette parole, d'après Tite-Live, ne sema pas seulement la terreur au sein du peuple, mais aussi parmi les sénateurs. L'habile tribun Sextius se saisit de cette occasion pour réprimander très fortement le peuple romain, en affirmant: "L'entendez-vous, citoyens, menacer et traiter en esclaves ses soldats? Et c'est une brute pareille que vous alliez juger plus digne d'une haute charge que ceux qui vous offrent une ville et des terres en vous envoyant en qualité de colons, qui luttent pour vos intérêts contre des ennemis si féroces et orgueilleux! Pourquoi vous étonner alors qu'ils soient si rares, ceux qui veulent se consacrer à la défense de votre cause! Qu'auraient-ils à espérer de vous? Des honneurs? Vous les réservez aux ennemis plutôt qu'aux amis du peuple romain. Vous avez gémi tout à l'heure en l'entendant parler. À quoi bon! Si en donnant votre vote vous préférez cet homme qui vous a si ignominieusement outragés, à ceux qui tiennent à vous procurer des terres, des logis, la fortune $!^{10}$ ”

3 Il est évident que ce récit est parsemé d'anachronismes. [...] Il est toutefois accessoire, pour la pertinence de mon propos, que le contexte du $V^{\mathrm{e}}$ siècle ne puisse ici être saisi de manière authentique. L'essentiel réside dans le fait que Tite-Live décrit des modèles de comportements qu'il n'aurait pas pu présenter au public de cette manière s'ils n'avaient pas correspondu au champ d'expérience et à l'horizon d'attente du lecteur ou de l'auditeur. 
Ce que le tribun M. Sextius, selon Tite-Live, reproche aux citoyens qui assistaient à l'assemblée qu'il a convoquée réside dans le fait que ces derniers, lors des élections des magistrats, ne se rallient pas à celui qui leur promet des avantages matériels, mais choisissent au contraire inlassablement les mêmes, même s'ils se sont comportés de manière inappropriée. Selon Sextius, les activités des magistrats dans leurs ressorts n'avaient qu'une influence réduite sur le comportement électoral du peuple dans les comices électoraux. Le peuple romain ne faisait qu'un usage très limité de ses possibilités de choix. Certains critères de sélection qui nous paraîtraient naturels dans l'appréciation portée sur les candidats, comme la capacité à défendre une politique en faveur du peuple, ne jouaient en tout cas pas un rôle important, tandis que certains éléments traditionnels, comme - la liste est loin d'être exhaustive - un nom (de famille) important et l'appartenance à la classe dirigeante, sont primordiaux ${ }^{11}$. Ce qui se relève ici très distinctement, s'agissant des assemblées populaires romaines, c'est l'écart important entre le pouvoir de décision formel et l'utilisation effective de cette liberté de choisir dans le sens de ses propres intérêts élémentaires ${ }^{12}$. [...]

5 On peut naturellement se demander par quels moyens a été obtenue cette limitation de fait des marges de décision, laquelle rendit possible un degré élevé de continuité des élites et de stabilité sociopolitique. Sur cette question également, l'épisode décrit précédemment fournit quelques indications. Le tribun Sextius prétend que le peuple, dans son obstination, choisira malgré tout Postumius, bien qu'il ait offensé les citoyens par son arrogance. Pourtant, comme l'indique plus loin Tite-Live, les patres apprécient bien différemment la situation. Ils s'attendent à ce que le dérapage de Postumius puisse conduire les électeurs à une réaction contre les candidats de l'aristocratie et empêchent pour cette raison les potentiels concurrents de se présenter aux élections ${ }^{13}$. Les sénateurs, par conséquent, redoutent précisément ce que Sextius, lui, espère et entend encourager par son discours incendiaire, à savoir que le peuple rompe avec son comportement électoral habituel. Nous constatons ainsi que l'adhésion du peuple aux candidats de l'aristocratie constitue certes la norme, mais que cet attachement peut être perturbé, notamment, en l'espèce, lorsqu'un membre des strates dirigeantes a mal traité le peuple. Par conséquent, le rapport que les représentants de l'aristocratie entretiennent avec le peuple est essentiel pour obtenir la docilité de ce dernier.

6 À Rome, la domination de fait de l'aristocratie appelait ainsi une certaine conduite visà-vis du peuple, pour laquelle je souhaiterais utiliser le terme de « jovialité ». Je désigne ainsi une forme de relation entre des personnes d'un rang social différent, dans laquelle le plus puissant s'abstient de faire jouer son pouvoir et fait mine, au contraire, de se trouver au même niveau que son interlocuteur. Dans le même temps, les deux parties ont conscience de l'asymétrie sociale qui caractérise la relation. L'effet de la jovialité consiste non pas en ce qu'elle abolit la conscience de cette asymétrie, mais qu'elle en atténue la prégnance effective dans les différentes situations concrètes.

$7 \quad$ Le cœur du concept dont il est question ici réside dans l'écart entre les partenaires de la communication, et cette notion de " jovialité » est moderne, bien que le terme soit évidemment issu du latin iovialis. Iovialis n'apparaît qu'à l'époque impériale, où il revêt le sens tout à fait élémentaire de "relatif à Jupiter ». La plus ancienne occurrence provient de l'œuvre d'Arnobe, vers 300 ap. J.- $\mathrm{C}^{14}$. Ce n'est que durant le Moyen Âge chrétien, paradoxalement, que le mot acquiert une plus grande consistance. Celui qui est né sous le signe de Jupiter est alors désigné par le terme de iovialis, et on lui attribue comme qualité, dans l'astrologie médiévale, une certaine gaieté. 
8 Parmi les termes antiques qui se rapprochent de la jovialité dans le sens défini cidessus, comitas et civilitas sont les plus importants. La civilitas constitue une qualité centrale du bon princeps et peut parfaitement être traduite par "jovialité ». Ce mot n'apparaît toutefois qu'à l'époque impériale ${ }^{15}$, et il ne paraît pas judicieux de le reporter dans le contexte républicain, car on suggérerait ainsi que ce terme est proche du langage des sources de l'époque républicaine. Le terme comitas, quant à lui, est certes largement attesté dès l'époque républicaine et s'avère très proche de la jovialité ${ }^{16}$; cependant, il n'évoque pas, avec la netteté requise, l'inégalité sociale qui fonde la spécificité des relations de communication façonnées par la jovialité, si bien que l'élément fondamental de la notion est ainsi éludé ${ }^{17}$. Il est par conséquent recommandé d'introduire, pour circonscrire ce type de comportement, la notion moderne de « jovialité ».

9 Ainsi, lorsqu'un aristocrate romain rencontrait un membre d'un groupe social plus modeste, dans un contexte public ou semi-public, on lui demandait de se montrer jovial. Lors de la salutatio, le salut matinal adressé au patron dans sa maison, les visiteurs pouvaient attendre de leur hôte une certaine affabilité ${ }^{18}$. Lors d'une campagne, le candidat devait à tout le moins affecter d'entretenir, avec les hommes qu'il rencontrait sur le forum, une relation personnelle, en les appelant par leurs noms ; pour ce faire, à la fin de la République, à l'heure où Rome était devenue une immense cité, il était indispensable de s'entourer de nomenclatores, des esclaves possédant une bonne mémoire et capables de souffler aux prétendants les noms des personnes rencontrées $^{19}$. Le magistrat devait ensuite franchir physiquement la distance qui le sépare des électeurs, en serrant la main des passants. [...]

10 Lors de la campagne, chaque candidat répondait personnellement de ses erreurs, sans que la propension fondamentale du peuple à suivre les recommandations des sénateurs ne fût mise en péril ${ }^{20}$. Il en allait autrement dans les assemblées populaires officielles, au sein desquelles un sénateur assumait le rôle de représentant de la politique du Sénat et où une entorse au code de conduite pouvait remettre en cause la prééminence de l'ordre sénatorial tout entier. L'enjeu était donc, pour le Sénat, que chacun maîtrisât le répertoire standard du rapport au peuple. Ce fut ainsi une construction avantageuse que seuls ceux qui avaient montré lors de la campagne qu'ils savaient faire preuve d'une certaine jovialité fussent élus et appelés à assumer les fonctions essentielles à la préservation des rapports existants. Cela ne se passait pas toujours bien, comme le montre l'exemple de Postumius évoqué précédemment. Postumius, justement, n'était pas sorti de son rôle en tant que candidat et ainsi en son nom propre, mais en tant que tribun consulaire en exercice, qui, en tant que représentant officiel du régime aristocratique, s'opposait au projet de colonie d'un tribun de la plèbe ; il ne mettait pas ainsi en péril sa propre réélection, dont il n'était aucunement question, mais l'approbation routinière des candidats de l'aristocratie.

11 Il est cohérent avec la vision que les Romains avaient ultérieurement de leur histoire qu'il ait fait partie des devoirs des magistrats supérieurs, dès le début de la République, d'adopter une attitude respectueuse vis-à-vis du peuple, de s'abstenir d'exhiber les pouvoirs de commandement dont sont revêtus les magistrats et de reconnaître le pouvoir de décision du peuple. Nous le percevons partout dans les assemblées populaires : les magistrats n'ont de cesse d'insister sur le fait que c'est au peuple qu'ils doivent la charge dont ils sont investi ${ }^{21}$; ils mettent également en exergue, d'une façon générale, les compétences décisionnelles du peuple ${ }^{22}$ et la libertas populi Romani ${ }^{23}$. Il ne 
s'agit aucunement d'une hypocrisie calculée, car l'hommage rendu à la liberté est tout à fait authentique, dans la mesure où il constitue l'exécution évidente de modes de comportement inscrits dans le rituel des assemblées populaires ${ }^{24}$. On ne saurait pour autant voir dans ce geste une preuve de l'ampleur des libertés fondamentales et en particulier du pouvoir de décision du peuple romain, dans la mesure où cette attitude de l'aristocratie répond à l'obéissance du peuple dans les affaires essentielles de l'État ${ }^{25}$. Celui qui, en tant que sénateur, se soumettait à l'exigence de jovialité à l'assemblée pouvait s'attendre à ce que le peuple suivît ses recommandations; et quand le peuple ne semblait pas vouloir prendre part au jeu, il pouvait être rappelé à l'ordre avec une ferme autorité. [...] La condition pour qu'une telle attitude fût acceptée résidait, il est vrai, dans la référence à la res publica ${ }^{26}$, qui appartenait bien au peuple ${ }^{27}$. Cette référence avait fait défaut dans le cas de Postumius.

Pour mieux comprendre pour quelles raisons le comportement de Postumius Regillensis dans l'épisode rapporté par Tite-Live, dont j'ai fait le fil rouge de la présente contribution, comporte d'aussi nombreuses conséquences, il convient d'examiner plus en détail les péchés dont s'est rendu coupable le tribun consulaire. Postumius s'était attiré l'hostilité de ses soldats, parce qu'il leur avait promis, avant la prise de Bolae, le pillage de la cité, mais n'avait pas respecté cet engagement une fois la cité prise ${ }^{28}$. Quand il fut ensuite rappelé pour lutter avec ses collègues contre les tribuns de la plèbe qui formaient le projet d'une loi de colonisation, il en vint à la menace formulée à l'encontre de ses soldats, qui suscita l'indignation générale. Sur ce point, il est intéressant de noter que le rude conflit de fond sur le projet d'implantation, soutenu par les tribuns de la plèbe, mais rejeté par les tribuns consulaires, ne constituait pas, semble-t-il, une réelle menace pour la domination de l'aristocratie. Dans les assemblées populaires, le Sénat et ses dignitaires pouvaient visiblement lutter contre des projets de loi très populaires sans que l'approbation docile apportée par le peuple aux candidats de l'aristocratie n'en pâtît. Ce n'est que par sa parole que Postumius transgresse véritablement les bonnes manières politiques, comme le souligne Tite-Live en indiquant que les propos incontrôlés du tribun consulaire avaient horrifié non seulement les soldats et le peuple - donc les soldats potentiels ${ }^{29}$-, mais également les patres, c'est-à-dire les sénateurs ${ }^{30}$. Les sénateurs ne sauraient assurément être suspectés de vouloir adoucir la discipline stricte régnant habituellement au sein de l'armée romaine et les peines sévères prévues en cas d'insubordination. Par ailleurs, la répartition du butin avec les soldats, en temps normal, ne leur tenait pas à cœur ${ }^{31}$. Le problème, du point de vue des sénateurs, réside moins dans ce que Postumius fait ou dans ce qu'il défend que dans la manière dont il se comporte devant le peuple.

Cette lecture à deux niveaux est aussi évidente que fondamentale. On peut décrire les assemblées populaires romaines comme des rituels publics, c'est-à-dire comme des cérémonies normées, centrées sur la répétition du scénario et marquées par une dimension symbolique forte. Bien entendu, elles avaient aussi une fonction instrumentale ${ }^{32}$, puisqu'elles servaient immédiatement à informer les citoyens et à prendre des décisions sur des sujets très divers et variables. Toutefois, à travers leur imbrication avec le sacré, l'habitus des magistrats et des sénateurs arborant les emblèmes associés à leur dignité, la forme topique des discours et la formation des unités de vote lors des assemblées délibératives, ces réunions comportaient aussi une forte charge symbolique, destinée à valoriser la communauté - naturellement, non pas une communauté d'égaux, mais une communauté tout à fait inégalitaire ${ }^{33}$. 
14 L'erreur commise par Postumius s'établissait sur le plan de la symbolique de la communauté, et non sur le plan instrumental et du contenu. C'est dans une autre catégorie que l'on doit ranger le comportement du tribun M. Sextius, qui, dans l'épisode que j'ai pris comme fil rouge, représente l'antithèse de Postumius, non seulement au niveau de l'intrigue, mais aussi dans la manière dont il démontre, en quelque sorte sous l'angle opposé, que le caractère institutionnel des relations entre strates supérieures et strates inférieures qui se concrétise dans les rituels publics est parfaitement susceptible de se dérégler. La désaffection du peuple vis-à-vis de l'aristocratie, cette menace pour l'équilibre du système, combattue au moyen de toutes les nuances du mos maiorum, peut être accentuée non seulement par la voie qu'incarne une figure comme celle de Postumius, c'est-à-dire par une attitude arrogante et un manque de jovialité à l'égard du peuple. La figure de Sextius incarne une autre voie du délitement. Quand il déclare, dans le discours que lui prête Tite-Live, que les hommes politiques en activité auraient perdu l'envie de défendre le partage des terres entre autres, parce qu'ils ne seraient de toute façon pas récompensés par le peuple lors des élections aux magistratures supérieures ${ }^{34}$, il définit ainsi une tactique politique, consistant à utiliser des lois accommodantes au service de sa propre carrière et ainsi arracher le peuple à son consentement docile.

15 Cette tactique constitue une composante dominante de la politique tribunicienne dans le récit livien de la période du conflit des ordres. C. Licinius Stolo et L. Sextius Lateranus, les protagonistes des lois licinio-sextiennes, au moyen desquelles, en 367-366, un certain équilibre fut obtenu entre patriciens et plébéiens, se plaignaient eux aussi, selon Tite-Live, que la plèbe fût favorable à ce que les tribuns de la plèbe luttassent pour la remise des dettes et contre l'occupation illimitée de l'ager publicus, mais fissent preuve d'indifférence quand il s'agissait de désigner ses champions aux magistratures supérieures ${ }^{35}$. Comme j'ai déjà pu le remarquer, ce témoignage n'apporte aucun élément fiable au sujet des motivations et des lignes de conflit aux premiers temps de la République, mais il est très révélateur que dans l'annalistique, à laquelle Tite-Live doit ces récits et même leur tonalité, l'on tienne pour évident que les tribuns de la plèbe sont animés du désir de voir leur engagement pour la plèbe récompensé par une élection aux magistratures supérieures. C'est dans cette optique, visiblement, que l'on envisageait, à la fin de la République - et peut-être même à une époque antérieure -, les activités populaires des tribuns. [...]

16 Ce lien entre l'engagement associé à la fonction de tribun de la plèbe de défendre les intérêts du peuple et l'espoir d'acquérir, par la prise en charge de ces missions, de la popularité et du support, et d'en tirer profit pour la suite de sa carrière de magistrat était malgré tout, au final, très étroit ${ }^{36}$. Cela ne signifie aucunement que les tribuns de la plèbe dussent se positionner en opposition systématique à l'establishment sénatorial. Ils n'avaient pas en permanence des intérêts du peuple à défendre à la satisfaction desquels le Sénat entendait s'opposer, d'autant plus qu'une large part de ces intérêts concernait le maintien et la protection des libertés civiques traditionnelles, dont le respect, pour les magistrats et les sénateurs, faisait de toute façon partie de la posture conventionnelle de jovialité. Dans ce domaine, les tribuns de la plèbe avaient souvent affaire aux dénonciations et, le cas échéant, aux poursuites engagées contre des hommes, qui, parce qu'ils étaient contrevenus à l'impératif de jovialité ou avaient commis des actes arbitraires en matière de politique extérieure, avaient également été quelque peu marginalisés au sein du Sénat ${ }^{37}$. Sur la durée, il était toutefois inévitable 
que des oppositions se fissent jour. La raison d'être et l'histoire du tribunat de la plèbe, en vertu desquelles il devait surtout garantir, contre les magistrats, les droits acquis graduellement par la masse du peuple ${ }^{38}$, le plaçaient structurellement en opposition aux magistrats curules et donc au Sénat qu'ils représentaient en principe, et l'on pouvait ainsi logiquement s'attendre à un certain nombre de conflits. En intégrant le tribunat de la plèbe, on avait aussi intégré ce potentiel de conflits inhérent à la structure. Aussi cette intégration dans l'État n'aurait-elle pu être obtenue sans la formation d'une remarquable tolérance aux écarts de la part de la classe politique dirigeante, qui devait, mais pouvait aussi s'accommoder du fait que la jeune génération d'hommes politiques, en tant que tribuns de la plèbe, agisse à l'occasion en faveur du peuple d'une manière fâcheuse pour le Sénat. C'est seulement lorsqu'une telle attitude était combattue en situation, mais dans l'ensemble acceptée et digérée sans que la fonction fût délégitimée ou que ses titulaires fussent proscrits ou poursuivis que le tribunat de la plèbe pouvait aussi assumer la fonction de soupape que Cicéron lui attribue explicitement ${ }^{39}$.

17 Dans son traité De legibus, sans doute élaboré en 51 av. J.-C. et conçu comme un dialogue entre des personnalités contemporaines, Cicéron prête à son frère Quintus de virulentes attaques contre le rôle du tribunat de la plèbe dans la vie publique romaine, auxquelles il répond ensuite lui-même. Cicéron concède certes parfaitement au Quintus du dialogue qu'il a pu y avoir beaucoup de mauvais tribuns de la plèbe, mais il estime que cette fonction est dans l'ensemble parvenue à faire en sorte que l'ordre le plus élevé ne fasse plus l'objet de jalousie et que le peuple ne lutte plus pour ses droits d'une manière dangereuse ${ }^{40}$. Au demeurant, dans un collège de dix hommes, il y en a selon lui toujours un, normalement, qui fait preuve de bon sens ${ }^{41}$. [...] Sur ce point, Cicéron s'attend ainsi à ce qu'au moins un tribun par an partage ses convictions et bloque par son droit de veto, le cas échéant, les initiatives déviantes de ses collègues ${ }^{42}$.

Dans l'ensemble, Cicéron, dans ses traités de théorie politique, attribue au tribunat de la plèbe un impact tout à fait positif sur la res publica ${ }^{43}$. De ce point de vue, les descriptions de Cicéron sur la manière dont cet effet a selon lui été obtenu sont particulièrement intéressantes. Au plus fort de la crise, à l'heure où la plèbe avait déjà pris les armes, les Anciens auraient agi de manière très sage en accordant à la plèbe le tribunat de la plèbe. Non seulement la seditio, autrement dit l'affrontement conduisant aux guerres civiles, a ainsi été surmontée, mais on a aussi trouvé par là une mesure modérée, par laquelle les petites gens pouvaient se sentir du même niveau que les principes, situation dans laquelle a nécessairement résidé, selon lui, le salut de l'État ${ }^{44}$. Nous comprenons ainsi clairement, chez Cicéron, que la jovialité revêtait la plus grande importance pour le fonctionnement de l'État romain. Les tribuns de la plèbe sont pour ainsi dire les gardiens de la jovialité. D'une certaine manière, il leur revenait de faire en sorte que, dans certaines situations spécifiques du rapport au peuple, la distance fût symboliquement aplanie, sans être réellement abolie. Par suite, le peuple se sentait placé, d'une certaine façon et dans un certain contexte, au même niveau que les principes, et cela suffisait à ce que la lutte pour l'égalité effective n'eût plus à être menée.

19 Cicéron poursuit ensuite l'analyse en suggérant que, sans cette jovialité institutionnalisée, on n'aurait pas pu chasser les rois ou bien on aurait dû donner au peuple la libertas non pas en parole, mais réellement. Cette liberté aurait toutefois ainsi été accordée qu'elle aurait été reconnue par de nombreux hommes de renom afin de la 
soumettre à l'auctoritas des principes ${ }^{45}$. Dans son traité sur la République, Cicéron l'affirme plus nettement encore, en évoquant les réalisations du premier consul Valerius Publicola. Ce dernier, selon lui, n'était pas du tout un homme médiocre, car, en accordant au peuple une liberté modérée, il n'en a que plus facilement préservé l'auctoritas des principes ${ }^{46}$. Dans le diagnostic de Cicéron, la libertas du peuple, limitée de telle sorte, en fin de compte, qu'elle était soumise à l'auctoritas des dirigeants, constitue ainsi davantage un élément formel qu'un élément substantiel ${ }^{47}$. L'accent mis sur la libertas à travers les mots et les gestes, qui constitue une composante essentielle de l'attitude joviale des membres des strates supérieures vis-à-vis du peuple, permettait de veiller à ce que ce dernier, en temps normal, ne fit pas usage des possibilités de choix dont il disposait formellement, mais suivît au contraire les principes. [...]

Dans le récit évoquant le tribun consulaire Postumius Regillensis et son adversaire, le tribun de la plèbe M. Sextius, se dessinent deux modèles de comportements déviants, qui sommeillaient dans la culture politique complexe de la res publica romaine: le contrôle du peuple au moyen de lois accommodantes, que je souhaiterais appeler ici "la déviance de type Sextius ", et l'irritation du peuple, provoquée par un manquement à l'impératif de jovialité, que je désignerai, par analogie, comme la « déviance de type Postumius ». Ces deux modèles ne semblent pas avoir été aussi dangereux l'un que l'autre pour l'oligarchie romaine. Comme le montrent tout à fait clairement les reproches que le tribun de la plèbe adresse au peuple sur son comportement électoral, les seules lois accommodantes ne suffisent pas à mettre à mal la tradition selon laquelle le peuple suit les décisions recommandées par la classe dirigeante établie ; autrement dit, le modèle de la déviance de type Sextius, qui se place à un niveau instrumental, est certes en lui-même fâcheux, mais ne constitue pas une menace fondamentale. On ne pouvait empêcher qu'à la longue des situations apparussent à la faveur desquelles des hommes politiques de la jeune génération parvenaient à se distinguer à l'aide des prérogatives associées au tribunat du peuple et saisissait la balle au bond. Dans l'ensemble, l'oligarchie pouvait bien supporter des confrontations intermittentes entre les tribuns de la plèbe et des représentants de la politique sénatoriale, et elle a ainsi développé sur ce point une remarquable tolérance aux écarts. Dans l'épisode que j'ai retenu comme fil rouge, ce n'est par conséquent qu'au moment où le représentant de la classe dirigeante enfreint grossièrement la civilité d'usage et brandit comme une menace, dans une assemblée populaire, le pouvoir qu'il détient, en tant que commandant, de punir sévèrement ses troupes ${ }^{48}$, que la docilité fondamentale du peuple est sensiblement perturbée. Chez les soldats, cette attitude mène à l'excès de violence que représente la lapidation ${ }^{49}$; pour les civils, à Rome, elle conduit à ce que l'on ne soit plus disposé d'office à élire les candidats de l'aristocratie. Le schéma de la déviance de type Postumius, autrement dit lorsque la communauté, habituellement produite et renforcée par la symbolisation de gestes de jovialité, est divisée par la démonstration grossière d'un pouvoir autoritaire, s'avère de toute évidence plus dangereux que le schéma de la déviance de type Sextius ; la structure institutionnelle de la République romaine, en d'autres termes, était avant tout stabilisée par des actes symboliques.

21 Toutefois, seule la rencontre de ces deux types de déviances semble avoir recelé une puissance explosive déterminante. Cela pourrait expliquer le fait que la validité des normes traditionnelles, malgré un nombre relativement important d'entorses individuelles aux comportements attendus, ne s'est pas fondamentalement affaiblie sous la République romaine. Aussi longtemps qu'un équilibre put se maintenir dans les 
assemblées populaires entre la fonction des tribuns de la plèbe - la sauvegarde des droits fondamentaux du peuple ${ }^{50}$ - et la fonction des représentants du Sénat - le contrôle de la communauté par la classe dirigeante, aussi longtemps, donc, que les déviances de type Sextius et de type Postumius ne se cumulèrent pas dans ces rituels publics, on put supporter de nombreux écarts individuels. C'est par ce schéma, peutêtre, que l'on peut aussi comprendre ce qui a mal fonctionné à la fin de la République. Les formes politiques du dernier siècle de la République, que nous définissons à partir de l'opposition cicéronienne entre populares et optimates, sont en écart par rapport aux normes de la res publica traditionnelle; la méthode utilisée par les populares correspondant à ce que j'ai appelé la déviance de type Sextius, cependant que la réaction des optimates renvoie aux déviances de type Postumius. Si ces déviances de type Sextius se firent plus marquées et plus fréquentes, je ne pense pourtant pas que l'on n'ait pas pu s'en accommoder. Les déviances de type Postumius, de surcroît en partie glorifiées comme une attitude adaptée, étaient plus graves. Une crispation se fit jour dans la gestion de la politique populaire par la classe dirigeante sénatoriale, pour reprendre la formule de Christian Meier ${ }^{51}$. De la part du Sénat, s'opposer résolument aux déviances de type Sextius faisait assurément partie d'une convention; dès l'épisode des Gracques, cependant, il semble que l'on ait négligé le principe selon lequel il fallait, pour préserver la docilité du plus grand nombre, reconnaître dans les mots la libertas qui signifiait aussi la souveraineté théorique du peuple - et ne pas l'offenser grossièrement, ce qui impliquait notamment d'argumenter dans l'espace public. À la place de cette attitude, le rejet obstiné semble être devenu une stratégie pour les optimates : on évite de débattre au Sénat des projets populaires ${ }^{52}$ et on éconduit parfois gentiment le peuple, dans les assemblées, au motif sommaire qu'il ne fallait rien changer ${ }^{53}$. Le refus de justifier l'opposition aux demandes populaires en se référant au bien de l'État et ainsi de prendre au sérieux les aspirations du peuple constitue clairement une déviance de type Postumius, une infraction à l'impératif de jovialité ${ }^{54}$.

Ce procédé nous apparaît de manière clairement évidente dans l'épisode survenu en 59, lorsque César, alors consul, présenta devant l'assemblée sa première loi agraire, dont le Sénat ne voulait pas débattre, et laissa également la parole à son collègue Bibulus. Ce dernier s'étant borné à répondre, contre ce projet qui avait reçu un large soutien à l'assemblée, qu'il ne souffrirait aucun changement durant son consulat, César continua d'attiser l'atmosphère, en affirmant que cette loi agraire se concrétiserait si Bibulus le voulait ${ }^{55}$; quand les participants pressent alors Bibulus de s'incliner, ce dernier s'exclame : « Vous n'aurez pas cette loi cette année, quand bien même vous la voudriez tous $!^{56}$ » Le peuple fut indigné par cette inacceptable déviance de type Postumius, et à juste titre, au regard des normes traditionnelles : au lieu de se montrer jovial, comme on l'attend d'un magistrat supérieur se présentant devant l'assemblée, et de reconnaître sur le principe le pouvoir de décision formel du peuple, Bibulus avait souligné ouvertement et avec mépris la prédominance matérielle de la classe dirigeante. Ce n'est assurément pas un hasard si, en tentant de faire obstacle, avec quelques tribuns de la plèbe, au vote de la loi, dont le jour avait été fixé, Bibulus vit ses faisceaux brisés ${ }^{57}$. L'étalage du pouvoir de coercition que symbolisent les faisceaux, totalement inapproprié dans le contexte d'une assemblée populaire, fut ainsi concrètement sanctionnés8 ${ }^{5}$. De même, le fait que l'on vida un panier de fumier sur la tête de Bibulus $^{59}$ doit être interprété de manière symbolique: par cet acte d'humiliation, le consul, qui s'était refusé à faire montre de jovialité à l'assemblée et avait préféré afficher sa propre supériorité, se vit ostensiblement refuser le respect 
implicitement dû à sa supériorité. Dans les déviances de type Postumius dont Bibulus se rendit coupable se relève très clairement l'échec des optimates dans le recours aux déviances de type Sextius.

L'épisode soulève pourtant une autre question ${ }^{60}$. En soutenant ou en prétendant soutenir les demandes populaires contre son homologue et contre le Sénat, le consul César contribua à une confusion des rôles traditionnels : le comportement de César correspondait à une déviance de type Sextius, ainsi commise par un consul, et non par des tribuns ${ }^{61}$. Les lignes qui se formaient de manière presque automatique lorsque survenaient les types de déviance habituels devenaient ainsi confuses, l'équilibre relatif qui prévalait dans les discussions exigibles lors des conflits était ainsi rompu ${ }^{62}$. C'est là aussi un symptôme du délitement de la République.

Le geste de la jovialité ne perdit pas en importance avec la mise en place du Principat augustéen. De même qu'un sénateur républicain était tenu, dans les champs institués de la communication publique, de traiter le peuple avec une déférence et une prévenance qui reléguaient au second plan les rapports de force réels, plutôt que de les exhiber, de même l'empereur, à Rome, ne devait pas montrer et encore moins souligner son pouvoir de domination; il devait au contraire se comporter, du moins vis-à-vis des sénateurs, comme un primus inter pares. Assumer cette forme spécifique de jovialité n'était déjà pas une mince affaire au temps de la République, de sorte que la déviance de type Postumius constitua une tentation constante ; mais sous le Principat, à l'heure où le déséquilibre des forces s'amplifia démesurément alors même que le code des manières joviales parvint à se maintenir, le risque d'un écart par rapport aux normes s'accrut également. En définitive, il n'est ainsi guère étonnant que peu d'empereurs parvinrent à bien maîtriser ce rôle complexe, exigeant force discernement et discipline de soi. Auguste fut dans cet art un maître sans égal. Les mots par lesquels il prit congé de ses amis à l'article de la mort - du moins selon le récit de Suétone - laissent à penser qu'il avait conscience de l'importance centrale de ce jeu de rôle dans le Principat. Après leur avoir demandé s'ils pensaient qu'il avait bien joué le drame de la vie, il alla jusqu'à citer le vers final d'une comédie :

« Si la pièce

Vous a plu, donnez-lui vos applaudissements

Et, tous ensemble, manifestez-nous votre joie ${ }^{63}$. »

\section{BIBLIOGRAPHIE}

Badian, Ernst (1972) : « Tiberius Gracchus and the Beginning of the Roman Revolution », ANRW, I.

1, Berlin / New York, p. 668-731.

Badian, Ernst (1990) : « The Consuls, 179-49 BC », Chiron, 20, p. 371-413.

Bell, Andrew J. E. (1997) : «Cicero and the Spectacle of Power », JRS, 87, p. 1-22.

Bleicken, Jochen (1968) : Das Volkstribunat der klassischen Republik. Studien zu seiner Entwicklung zwischen 287 und 133 v. Chr., $2^{\mathrm{e}}$ éd., Munich. 
Bleicken, Jochen (1981) : « Das römische Volkstribunat Versuch einer Analyse seiner politischen Funktion in republikanischer Zeit », Chiron, 11, p. 87-108.

Bleicken, Jochen (1998) : Augustus. Eine Biographie, Berlin.

Brunt, P. A. (1988) : The Fall of the Roman Republic and Related Essays, Oxford.

Bunse, Robert (1998) : Das römische Oberamt in der frühen Republik und das Problem der

«Konsulartribunen », Trèves.

Burckhardt, Leonhard Alexander (1988) : Politische Strategien der Optimaten in der späten römischen Republik, Stuttgart.

Crusius, O. (1914) : « Ultima vox Augusti (Suet. II 99) », Philologus, 73, p. 320.

David, Jean-Michel (1993) : «Conformisme et transgression : à propos du tribunat de la plèbe à la fin de la République romaine », Klio, 75, p. 219-227.

Eder, Walter (1996) : « Republicans and Sinners. The Decline of the Roman Republic and the End of a Provisional Arrangement ", in : Wallace, Robert W. / Harris, Edward M. (éd.) : Transitions to Empire. Essays in Greco-Roman History, 360-146 B.C., in honor of E. Badian, Norman / Londres, p. 439-461.

Feig Vishnia, Rachel (1996) : State, Society and Popular Leaders in MidRepublican Rome 241-167 BC, Londres / New York.

Ferrary, Jean-Louis (1984) : «L'archéologie du De re publica $(2,2,4-37,63)$ : Cicéron entre Polybe et Platon ", JRS, 74, p. 87-98.

Flaig, Egon (1992) : Den Kaiser herausfordern. Die Usurpation im Römischen Reich, Francfort-sur-leMain / New York.

Flaig, Egon (1993) : « Politisierte Lebensführung und ästhetische Kultur. Eine semiotische Untersuchung am römischen Adel », Historische Anthropologie, 1, p. 193-217.

Flaig, Egon (1994) : «Repenser le politique dans la République romaine », Actes de la Recherche en Sciences sociales, 105, p. 13-25.

Flaig, Egon (1995) : « Entscheidung und Konsens. Zu den Feldern der politischen Kommunikation zwischen Aristokratie und Plebs ", in : Jehne, Martin (éd.) : Demokratie in Rom? Die Rolle des Volkes in der Politik der römischen Republik, Stuttgart, p. 77-127.

Flaig, Egon (1997) : « Zwingende Gesten in der römischen Politik », in : Chvojka, Erhard / Dülmen, Richard van / Jung, Vera (éd.) : Neue Blicke. Historische Anthropologie in der Praxis, Vienne, p. 33-50.

Flaig, Egon (1998) : « War die römische Volksversammlung ein Entscheidungsorgan? Institution und soziale Praxis », in : Reinhard Blänkner / Bemhard Jussen (éd.) : Institutionen und Ereignis. Über historische Praktiken und Vorstellungen gesellschaftlichen Ordnens, Göttingen, p. 49-73.

Fontanella, Francesca (1998) : «Introduzione al De legibus di Cicerone. II », Athenaeum, 86, p. 179-208.

Girardet, Klaus M. (1977) : « Ciceros Urteil über die Entstehung des Tribunates als Institution der römischen Verfassung (rep. 2,57-59) », in : Bonner Festgabe für Johannes Straub, Bonn, p. 179-200.

Goltz, Andreas (2000) : « Maiestas sine viribus - Die Bedeutung der Lictoren für die Konfliktbewältigungsstrategien römischer Magistrate », in : Linke, Bernhard / Stemmler, Michael (éd.) : Mos maiorum: Untersuchungen zu den Formen der Identitätsstiftung und Stabilisierung in der römischen Republik, Stuttgart, p. 237-267. 
Gugel, Helmut (1977) : Studien zur biographischen Technik Suetons, Vienne.

Hellegouarc'h, Joseph (1972) : Le vocabulaire des relations et des parties politiques sous la République, $2^{e}$ éd., Paris.

Hellmann, Fritz (1939) : Livius-Interpretationen, Berlin.

Heuer, Karl-Heinz (1941) : Comitas-facilitas-liberalitas. Studien zur gesellschaftlichen Kultur der ciceronischen Zeit, Münster (thèse).

Heuß, Alfred (1976) : «Ciceros Theorie vom römischen Staat », Nachrichten der Akademie der Wissenschaften in Göttingen, Philol.-hist. Kl., 8/1975, Göttingen, p. 193-272.

Heuß, Alfred (1983) : «Grenzen und Möglichkeiten einer politischen Biographie », HZ, 237, p. 85-98.

Hirzel, Rudolf (1967 [1909]) : Die Strafe der Steinigung, Darmstadt.

Hölkeskamp, Karl-Joachim (1990) : «Senat und Volkstribunat im frühen 3. Jh. v. Chr. », in : Eder, Walter (éd.) : Staat und Staatlichkeit in der frühen römischen Republik. Akten eines Symposiums 12.-15. Juli 1988 FU Berlin, Stuttgart, p. 437-457.

Hölkeskamp, Karl-Joachim (1993) : « Conquest, Competition and Consensus: Roman Expansion in Italy and the Rise of the Nobilitas ", Historia, 42, p. 12-39.

Hölkeskamp, Karl-Joachim (1995) : « Oratoris maxima scaena: Reden vor dem Volk in der politischen Kultur der Republik », in Jehne, Martin (éd.) : Demokratie in Rom? Die Rolle des Volkes in der Politik der römischen Republik, Stuttgart, p. 11-49.

Jehne, Martin (1995) : « Die Beeinflussung von Entscheidungen durch “Bestechung”: Zur Funktion des ambitus in der römischen Republik », in : id. (éd.) : Demokratie in Rom? Die Rolle des Volkes in der Politik der römischen Republik, Stuttgart, p. 51-76.

Kolendo, Jerzy (1989) : Nomenclator, « memoria » del suo padrone o del suo patrono. Studio storico ed epigrafico, Faenza.

Laser, Günter (1997) : Populo et scaenae serviendum est. Die Bedeutung der städtischen Masse in der Späten Römischen Republik, Trèves.

Libero, Loretana de (1992) : Obstruktion. Politische Praktiken im Senat und in der Volksversammlung der ausgehenden römischen Republik (70-49 v. Chr.), Stuttgart.

Lind, L. R. (1994) : « Thought, Life, and Literature at Rome: the Consolidation of Culture », in : Deroux, Carl (éd.) : Studies in Latin Literature and Roman History, VII, Bruxelles, p. 5-71.

Liou-Gille, Bernadette (1992) : «Le butin dans la Rome ancienne », in : La Rome des premiers siècles. Légende et histoire. Actes de la Table Ronde en l'honneur de Massimo Pallottino (Paris 3-4 Mai 1990), Florence, p. 155-172.

Meier, Christian (1980) : Res publica amissa. Eine Studie zu Verfassung und Geschichte der späten römischen Republik, $2^{\mathrm{e}}$ éd., Francfort-sur-le-Main.

Melville, Gert (éd.) (1997) : SFB 537 « Institutionalität und Geschichtlichkeit ». Ein neuer Sonderforschungsbereich stellt sich vor, TU Dresde.

Melville, Gert (1998) : «Institutionen im Mittelalter », Bulletin de la Société des Amis de l'Institut Historique Allemand Paris, 4, p. 11-33.

Millar, Fergus (1998) : The Crowd in Rome in the Late Republic, Ann Arbor.

Nippel, Wilfried (1988) : Aufruhr und «Polizei » in der römischen Republik, Stuttgart. 
Nippel, Wilfried (1997) : « Die plebs urbana und die politische Gewalt in der späten Republik im Spiegel der jüngeren französischen und deutschen Forschung ", in : Bruhns, Hinnerk / David, Jean-Michel / Nippel, Wilfried (éd.) : Die späte römische Republik / La fin de la République romaine, Rome, p. 237-252.

Oakley, S. P. (1997) : A Commentary on Livy Books VI-X, I : Introduction and Book VI, Oxford.

Perelli, Luciano (1979) : « Note sul tribunato della plebe nella riflessione ciceroniana », QS, 5, p. 285-303.

Richard, Jean-Claude (1990) : « Réflexions sur le tribunat consulaire », MEFRA, 102, p. 767-799.

Rilinger, Rolf (1982) : « Die Interpretation des Niedergangs der römischen Republik durch "Revolution" und "Krise ohne Alternative" ", AKG, 64, p. 279-306.

Rilinger, Rolf (1997) : « Domus und res publica. Die politisch-soziale Bedeutung des aristokratischen "Hauses" in der späten römischen Republik », in : Winterling, Aloys (éd.) : Zwischen « Haus » und «Staat ». Antike Höfe im Vergleich, Munich, p. 73-90.

Stylow, Armin U. (1972) : Libertas und Liberalitas. Untersuchungen zur innenpolitischen Propaganda der Römer, Munich (thèse).

Thommen, Lukas (1988) : « Das Bild vom Volkstribunat in Ciceros Schrift über die Gesetze », Chiron, 18, p. 357-375.

Ungern-Sternberg, Jürgen von (1973) : « Die popularen Beispiele in der Schrift des Auctor ad Herennium », Chiron, 3, p. 143-162.

Ungern-Sternberg, Jürgen von (1998) : « Die Legitimitätskrise der römischen Republik », HZ, 266, p. 607-624.

Vogt, Joseph (1978) : « Nomenclator. Vom Lautsprecher zum Namenverarbeiter », Gymnasium, 85, p. 327-338.

Wallace-Hadrill, Andrew (1982) : «Civilis princeps: Between Citizen and King », JRS, 72, p. 32-48.

Yakobson, Alexander (1995) : « Secret Ballot and its Effects in the Late Roman Republic », Hermes, 123, p. 426-442.

\section{NOTES}

1. Voir Melville (1997), p. 11 et suiv., p. 15.

2. Melville (1997), p. 11 et suiv.

3. L'idée d'une «crise sans alternative » développée par Meier en 1966 dans son étude sur la fin de la République romaine (Res publica amissa, $2^{\mathrm{e}}$ éd. 1980, en particulier p. 201-205), a été critiquée pour son caractère paradoxal. On lui a objecté également qu'une situation problématique qui laisse clairement entrevoir l'issue ne constitue pas une véritable crise (voir surtout Heuß [1983], p. 87 et suiv.). Par cette expression, Meier a voulu caractériser une configuration dans laquelle, malgré un sentiment de crise massif, jamais aucune alternative à l'ordre existant ne fut imaginée et dans laquelle aucune nouvelle couche ni aucun nouveau groupe ne s'apprêtait à prendre la relève des strates dominantes. Il n'en reste pas moins que la formule d'une crise sans alternative pose problème et appelle des explications (voir également Rilinger [1982], p. 290-292). La théorie des institutions permet peut-être de mieux comprendre ce phénomène. Selon cette théorie, la res publica romaine fut à travers les siècles un modèle institutionnel extraordinairement performant, qui, par son succès, habilement mis en scène, est devenu la condition indispensable du bien-être $\mathrm{du}$ peuple romain et du maintien de sa domination universelle. En d'autres termes, cette 
institution s'est établie comme l'unique possibilité pour mettre en œuvre les principes de sécurité, de domination et d'excellence de l'aristocratie romaine, au service de l'état et de la liberté du peuple romain. Le fait que l'on ne soit pas parvenu, à la fin de la République, à maîtriser le déséquilibre structurel entre, d'une part, les ambitions personnelles de certains personnages éminents dont la République ne pouvait se passer pour maintenir son emprise sur le monde méditerranéen et, d'autre part, la domination collective de la classe dirigeante révèle un conflit entre ces principes ; on éprouvait ce conflit avec force, mais on ne pouvait le résoudre. La res publica se montrait incapable de hiérarchiser ces principes. Eût-elle su le faire, elle aurait pris le risque de favoriser l'émergence de pouvoirs particuliers, ce que l'on entendait précisément éviter.

4. Voir notamment Badian (1990), p. 371-413.

5. Sur la compétition dans l'élite, voir Hölkeskamp (1993), p. 12-39.

6. Voir notamment Flaig (1992), p. 38-93, en particulier p. 76, 83-86 ; Flaig (1993), p. 197, p. 211 ; Flaig (1994), p. 13-25 ; Flaig (1995), p. 77-127 ; Flaig (1998), p. 52-58 ; ainsi que Bell (1997), p. 1-22. Sur la distinction entre "organisation formelle» et «interactions stéréotypées entre personnes », dans la perspective de la théorie des institutions, voir également Melville (1998), p. 15.

7. Tite-Live, 4, 49,7-50, 5. Une interprétation de ce passage à la lumière de la position et de la méthode de travail de Tite-Live figure chez Hellmann (1939), p. 41-46.

8. Tite-Live, 4, 49, 11 : [...] qua M. Sextio tribuno plebis legem agrariam ferenti, simul Bolas quoque ut mitterentur coloni laturum se dicenti - dignum enim esse qui armis cepissent, eorum urbem agrumque Bolanum esse - [...].

9. Tite-Live, 4, 49, 11 : "Malum quidem militibus meis" inquit, "nisi quieverint." Un autre exemple de menace de malheur se trouve chez Valère Maxime, 1, 6, 6. Alors que ses porte-enseignes déclaraient qu'on ne pouvait déplacer les signa, C. Flaminius les menace d'un malum, s'ils ne parviennent pas à arracher de terre les étendards. C'est ainsi que Flaminius engagea ses troupes dans la bataille du lac Trasimène et vers la catastrophe (voir également Cicéron, De divinatione, 1 , 77).

10. Tite-Live, 4, 49, 14-16: "Auditis" inquit, "Quirites, sicut servis malum minantem militibus? Tamen haec belua dignior vobis tanto honore videbitur quam qui vos urbe agrisque donatos in colonias mittunt, qui sedem senectuti vestrae prospiciunt, qui pro vestris commodis adversus tam crudeles superbosque adversarios depugnant? incipite deinde mirari cur pauci iam vestram suscipiant causam. Quid a vobis sperent? An honores, quos adversariis vestris potius quam populi Romani propugnatoribus datis? Ingemuistis modo voce huius audita. Quid id refert? Iam si suffragium detur, hunc qui malum vobis minatur, iis qui agros sedesque ac fortunas stabilire volunt praeferetis" (trad. Gérard Walter, coll. « La Pléiade »). Un passage presque identique, du point de vue de notre argumentation, se trouve chez Tite-Live, 4, 35, 5-11, pour le compte de l'année 424 ; mais voir surtout Rhétorique à Hérennius, 4, 48: "Miramini, Quirites, quod ab omnibus vestrae rationes deserantur? quod causam vestram nemo suscipiat? quod se nemo vestri defensorem profiteatur? Id tribuite vestrae culpae, desinite mirari. Quid est enim, quare non omnes istam rem fugere et vitare debeant? Recordamini, quod habueritis defensores; studia eorum vobis ante oculos proponite; deinde exitus omnium considerate. Tum vobis veniet in mentem, ut vere dicam, neglegentia vestra sive ignavia potius illos omnes ante oculos vestros trucidatos esse, inimicos eorum vestris suffragiis in amplissimum locum pervenisse." Ungern-Sternberg (1973), p. 158-162 formule l'hypothèse que le reproche selon lequel le peuple élirait ses ennemis fait concrètement allusion à Marcus Livius Drusus l'Ancien, consul en 112, et qu'il pourrait s'agir ici d'un extrait du discours effectivement tenu par C. Memmius, tribun en 111 (en raison d'un raisonnement similaire chez Salluste, Guerre de Jugurtha, 31, 1-3). Toutefois, ce grief me paraît constituer un élément d'un schéma argumentatif plus général, visant à critiquer la propension du peuple à élire des candidats de l'aristocratie, même si aucun exemple concret n'est directement présent (voir également Ungern-Sternberg [1973], p. 161). 
11. Voir Tite-Live, 4, 44, 2: Hoc tribuno comitia quaestorum habente, petentibusque inter aliquot plebeios filio $<A_{.}>$Antisti tribuni plebis et fratre alterius tribuni plebis Sex. Pompili, nec potestas nec suffragatio horum valuit quin quorum patres avosque consules viderant eos nobilitate praeferrent.

12. Comme l'a souligné à juste titre Bell (1997), p. 2, la puissance des assemblées se mesure aussi à la manière dont leur pouvoir de décision se réalise dans l'opposition, à travers le refus de légitimer l'ordre existant.

13. En veillant à ce que des consuls, et non des tribuns consulaires, soient élus (Tite-Live, 4,50 , $7-51,1)$. C'est un schéma courant chez Tite-Live que la décision de savoir si le consulat, encore réservé aux patriciens, ou bien le tribunat consulaire, également ouvert aux plébéiens, devait être établi comme la magistrature suprême de l'année suivante dépendait à chaque fois des discussions entre patriciens et plébéiens. Voir Bunse (1998), p. 101 et suiv., avec une série de références à la note 4 .

14. Arnobe, Contre les Gentils, 3, 40 ; 43 ; 5, 10 ; 21 ; 7, 21 ; voir aussi Martianus Capella, 1, 28 ; 35 ; $39 ; 40 ; 42 ; 94$; 2, 196 ; 208 ; 4, 424 ; 6, 582 ; 8, 806 ; 9, 892 ; 901 ; Macrobe, Saturnales, 5, 16, 10 ; Commentaire sur le songe de Scipion, 1, 19, 25 ; Boèce, Institution musicale, 1, 27 (p. 219, 8 Friedlein). L'indication donnée par Georges à l'entrée correspondante, selon laquelle une première occurrence figurerait déjà chez Apulée, est erronée, comme permet de le vérifier un contrôle dans le Thesaurus Linguae Latinae. Je tiens ici à remercier Dietfried Krömer (Thesaurus Linguae Latinae, Munich) pour son aide amicale pour les recherches dans le Thesaurus.

15. Voir Wallace-Hadrill (1982), p. 32-48, en particulier p. 42 et suiv.

16. Voir Heuer (1941), p. 24-45 ; Lind (1994), p. 17-19.

17. Heuer (1941), p. 27 et plus encore p. 29-33, considère toutefois l'écart social comme une composante de la notion de comitas (voir également Hellegouarc'h [1972], p. 215 et suiv.) et présente à l'appui de cette lecture de remarquables témoignages. Il doit pourtant concéder que l'asymétrie ne trouve pas encore de résonnance dans la comédie (Heuer [1941], p. 28 et suiv.), et il existe en outre d'autres exceptions (comme le lien avec l'amicitia dans le Laelius de Cicéron, qui ne présuppose aucun écart dans les relations, voir Laelius, 66). Ce n'est du reste pas étonnant, car c'est le propre des relations asymétriques d'être également occultées dans les notions, que l'on utilise d'emblée des termes confraternels dans une intention euphémistique (cf. l'évolution de la notion d'amicitia à la fin de la République : Brunt [1988], p. 360 et suiv.) ou que l'écart soit relégué au second plan dans les mots utilisés (sans toutefois s'effacer complètement).

18. Sur la salutatio, voir Rilinger (1997), en particulier p. 82-84.

19. Sur les nomenclatores, voir Vogt (1978), p. 327-338; Kolendo (1989). Voir aussi Jehne (1995), p. 58. Laser (1997), p. 129, note 220, critique mon hypothèse (Jehne [1995], p. 58, note 44), selon laquelle il s'agit d'entretenir la fiction d'une face-to-face-society (mais voir aussi, désormais, Millar [1998], p. 224 et suiv.). Dans l'article cité, mon propos n'était en effet pas clair. Dans cette communication directe entre le candidat et les électeurs, la composante fictive ne réside pas, naturellement, dans le geste de la poignée de main, mais dans l'adresse nominative, dans la mesure où le candidat, en fait, ne connaît pas les passants, ou du moins pas par leurs noms : avec l'aide des nomenclatores, qui étaient en même temps mal vus et incontournables, on affecte des relations personnelles.

20. Voir aussi [...] Valère Maxime, 7, 5, 2 : Igitur civitas nostra nobilium iuvenum ingenia ab insolentia revocando magnos et utiles cives fecit, honoribusque, non patiendo eos a securis peti, debitum auctoritatis pondus adiecit.

21. Sur la magistrature comme beneficium populi, voir notamment Cicéron, Discours sur la loi agraire, 2, 1-5.

22. Voir Cicéron, De oratore, 1, 225. Voir également Ungern-Sternberg (1998), p. 608 et suiv.

23. Cicéron, Discours sur la loi agraire, 2, 9 : Quid tam populare quam libertas ? Voir aussi Cicéron, Pro Sestio, 137. Sur les techniques de présentation dans les discours adressés au peuple, voir désormais Laser (1997), p. 143-182. 
24. Au sujet des hommes politiques populaires (populares), Nippel (1997), p. 240 et suiv. a souligné, fort justement et en s'en distanciant nettement, que la science allemande, en particulier, "a envisagé l'appel à la libertas et à la prospérité du peuple d'abord comme un élément d'une stratégie de manipulation ». Cette lecture est non seulement trop partielle pour les populares, mais elle ne permet pas, non plus, une juste évaluation du rapport des optimates à la libertas.

25. Voir par exemple Cicéron, Pro Sestio, 105, où il est tout d'abord indiqué que les graves et magni homines qui s'opposaient aux populares étaient impopulaires parmi le peuple, qui ne votait pas selon leur volonté. Toutefois, le passage s'achève par la phrase suivante : Ac tamen, si quae res erat maior, idem ille populus horum auctoritate maxime commovebatur. Voir aussi De legibus, 3, 39. Voir Laser (1997), p. 141, 149.

26. Voir également Hölkeskamp (1995), p. 39-41; Brunt (1988), p. 49 ; Laser (1997), p. 141 et suiv. Cf. Cicéron, De officiis, 1, 85 ; Post Reditum in Quirites, 1 ; Pro Sestio, 101-103 ; 105.

27. Voir la célèbre définition de Cicéron, De republica, 1, $39 ; 41$. Voir aussi Cicéron, De legibus, 3, 8, où il est demandé comme orientation particulière, pour les consuls notamment: Ollis [sc. consulibus] salus populi suprema lex esto.

28. Tite-Live, 4, 49, 9 et suiv. (avec une variante).

29. S'il s'agissait d'une menace dirigée contre les électeurs, comme l'a écrit Yakobson (1995), p. 427, cela déplacerait le sens. C'est plutôt l'inverse : Postumius menaça les soldats, encore à Bolae, et le peuple romain fut touché, parce que chacun pouvait un jour devenir soldat.

30. Tite-Live, 4, 49, 11 (à la suite du texte cité plus haut, note 8) : Quod auditum non contionem magis quam mox patres offendit. Comme l'explique Tite-Live dans la suite (\$12), les propos comme ceux de Postumius portaient non seulement préjudice à l'intéressé lui-même et à sa cause, mais aussi à l'ordre auquel il appartient dans son ensemble ([...] invidiae non ipsi tantum sed causae atque universi ordini [...]).

31. Sur les problèmes liés à la répartition du butin, voir par exemple Liou-Gille (1992), p. 155-172.

32. Les rituels présentent toujours, par essence, à la fois une dimension instrumentale et une dimension symbolique : voir par exemple Melville (1997), p. 11 ; Melville (1998), p. 22. Le critère de différenciation réside par conséquent dans le rapport de force entre ces composantes, ou plutôt dans la latitude offerte dans le champ des éléments instrumentaux, qui peut être importante (par exemple des organes de décision possédant une grande liberté quant au contenu, aux domaines et aux orientations des décisions) ou plus réduit (par exemple des organes d'enregistrement, qui se contentent de sanctionner ce qui est proposé, sans réelle possibilité de choix), ce qui induit peut-être un besoin de symbolique d'un niveau différent.

33. Voir Meier (1980), p. 52.

34. Tite-Live, 4, 49, 15 et suiv. (voir supra note 10).

35. Tite-Live, 6, 39, 10. D'autres témoignages à l'appui d'une telle argumentation figurent chez Oakley (1997), p. 693. Voir aussi Rhétorique à Hérennius, 4, 48 (supra note 10).

36. Voir également Bleicken (1981), notamment p. 100. David (1993), p. 219-227 adhère même à l'idée que la pression de se distinguer entraîne un mouvement de fond à s'écarter - de façon relativement minime il est vrai - des conventions.

37. Sur les poursuites engagées par des tribuns de la plèbe à l'égard des outsiders, visiblement en étroite collaboration avec la majorité du Sénat, voir Hölkeskamp (1990), p. 437-457; voir aussi Bleicken (1968), en particulier p. 137 et suiv.

38. Voir par exemple les témoignages réunis chez Bleicken (1981), p. 91, note 8 ; voir aussi Stylow (1972), p. 14 et suiv.

39. Voir également Meier (1980), p. 53.

40. Cicéron, De legibus, 3, 24 : Et praeter eos [les Gracques] quamvis enumeres multos licet, cum deni creantur, <non>nullos in omni memoria reperies perniciosos tribunos, leves etiam, non bonos, fortasse plures: invidia quidem summus ordo caret, plebes de suo iure periculosas contentiones nullas facit. 
41. Cicéron, De legibus, 3, 24 : Quod enim est tam desperatum collegium, in quo nemo e decem sana mente sit?

42. Voir également l'éloge explicite de ce comportement dans le catalogue des lois de Cicéron (De legibus, 3, 11) : Intercessor rei malae salutaris civis esto. Cicéron explique en 3, 43 : Quis non studiose rei publicae subvenerit hac tam praeclara legis voce laudatus?

43. Voir sur ce point Heuß (1976), p. 222-225 ; Girardet (1977), p. 179-200 ; Perelli (1979), p. 285-303 ; Bleicken (1981), p. 91 et suiv.; Ferrary (1984), en particulier p. 94-97; Thommen (1988), p. 357-375 ; Feig Vishnia (1996), p. 201 et suiv. ; Fontanella (1998), p. 205-207. Brunt (1988), p. 324-326 ne voit dans la lecture de Cicéron que le point de vue de classe cynique de l'aristocratie, qui n'aurait aucunement été partagé largement.

44. Cicéron, De legibus, 3, 24 : Sed tu sapientiam maiorum in illo vide : concessa plebei a patribus ista potestate, arma ceciderunt, restincta seditio est, inventum est temperamentum, quo tenuiores cum principibus aequari se putarent, in quo uno fuit civitatis salus.

45. Cicéron, De legibus, 3, 25 (à la suite du texte cité supra, note 44): Quam ob rem aut exigendi reges non fuerunt, aut plebi re, non verbo, danda libertas. Quae tamen sic data est, ut multis praeclarissimis addiceretur, ut auctoritati principum cederet. La leçon citée ici (et traduite dans le texte), ut multis praeclarissimis addiceretur, est celle qui est transmise dans les meilleurs manuscrits, tandis que les deteriores proposent adduceretur, en lieu et place d'addiceretur. Dans l'apparat critique de son édition (Berlin, 1883, $2^{\mathrm{e}}$ éd.), J. Vahlen a proposé ut multis <institutis> praeclarissimis adduceretur, leçon qui a cours, depuis lors, dans les différentes éditions (voir par exemple les éditions de

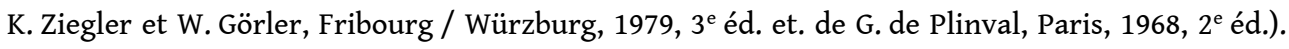
Dans leur édition (Francfort-sur-le-Main, 1824, rééd. Hildesheim, 1973), I. Davisius et G. H. Moser proposent: [...] ut multis SCtis praeclarissimis adduceretur (plusieurs autres propositions sont formulées dans l'apparat critique [p. 429]). Dans la version émendée d'après Vahlen, il faut comprendre que le sujet d'adduceretur (et de cederet) est bien le terme plebs, mentionné précédemment (terme que Ferrary [1984], p. 97, note 67 ajoute logiquement afin de clarifier le sens). Il serait logique, du point de vue de la syntaxe, que le sujet de la proposition introduite par quae soit aussi le sujet de la proposition introduite par ut qui en dépend; cette lecture est aisément confortée par le texte quand on le conserve tel qu'il est proposé dans les meilleurs manuscrits (il en va naturellement ainsi, également, chez Perelli [1979], p. 297). Dans une paraphrase visant à expliciter le texte, on peut en restituer le contenu comme suit : la libertas a ainsi été donnée qu'elle a été à maintes reprises accordée au peuple par beaucoup d'hommes très renommés, afin que la libertas se soumette, en dernière instance, à l'auctoritas des principes. Ainsi, Cicéron semble bien décrire ici les appels à la libertas que les magnats formulent devant le peuple comme un moyen de domination.

46. Cicéron, De republica, 2, 55 : Haud mediocris hic ut ego quidem intellego vir fuit [Publicola], qui modica libertate populo data facilius tenuit auctoritatem principum. Voir également Plutarque, Publicola, 10, 7-9.

47. Voir Fontanella (1998), p. $206:$ : [...] più apparente che reale ».

48. Il est très probable que la parole de Postumius renvoie à la menace de sévères mesures disciplinaires. Lorsque le questeur de Postumius entend effectivement agir contre les soldats frondeurs, il est blessé par un jet de pierre et mis en fuite, le coupable lui faisant savoir que le questeur ne faisait que subir ce dont le commandant les avait menacés (Tite-Live, 4, 50, 3). Si l'on retranscrit ce propos littéralement, les soldats voyaient ainsi derrière la menace de Postumius des châtiments corporels, voire des exécutions, que Postumius tenta ensuite effectivement d'imposer peu avant sa mort (Tite-Live, 4, 50, 4 et suiv.). Que Postumius fut tué pour avoir trompé ses soldats lors de la répartition du butin (ainsi Richard [1990], p. 796) constitue une simplification schématique du récit de Tite-Live.

49. L'importante charge symbolique que véhicule la lapidation dans l'environnement grec, où elle symbolise l'expulsion hors de la communauté, ne semble pas avoir prévalu en contexte 
romain, du moins selon Hirzel (1967 [1909]), p. 258 et suiv. Voir cependant Flaig (1993), p. 195 et suiv.

50. Voir Brunt (1988), p. 324 et suiv. ainsi que la note 109.

51. Meier (1980), p. 129, note 400. La ferme intercessio du tribun M. Octavius, en 133, participe également de cette crispation (voir Badian [1972], p. 706-711; voir aussi Eder [1996], p. 447). Sur les tentatives de Tiberius Gracchus pour dissuader Octavius de faire valoir son veto et le refus obstiné d'Octavius d'introduire des compromis dans les rituels en vigueur, voir Flaig (1997), p. 33-39.

52. Voir le chapitre « mesures dilatoires » chez de Libero (1992), p. 15-28.

53. Ainsi Quintus Lutatius Catulus en 67 (Cicéron, De Imperio Cn. Pompei, 59 et suiv.) et Bibulus en 59 (Dion Cassius, 38, 4, 3, de même que Caton au Senat en 59 : Dion Cassius, 38, 3, 1).

54. Burckhardt (1988) parvient dans son étude à la conclusion que la politique des optimates visait en premier lieu le blocage, c'est-à-dire consistait à empêcher la résolution des problèmes urgents et la satisfaction des intérêts d'autres groupes. Pour ce faire, ils élargissaient notamment des règles procédurales, rivalisaient de démagogie ou affaiblissaient insidieusement le contenu des mesures réformatrices (voir également Ungern-Sternberg [1998], p. 614-622). La déviance de type Postumius représente typologiquement une autre facette de la crispation des optimates.

55. Dion Cassius, 38, 4, 3.

56. Dion Cassius, 38, 4, 3 (trad. Guy Lachenaud et Marianne Coudry, coll. « CUF »).

57. Dion Cassius, 38, 6, 3 ; Plutarque, Pompée, 48,1 ; Caton le Jeune, 32, 4 ; Appien, Guerres civiles, 2 , 11 (38).

58. Sur les cas évoquant cet acte ostensiblement symbolique qui nous sont parvenus, voir Nippel (1988), p. 24, 62 et 120 ; voir aussi Goltz (2000).

59. Plutarque, Pompée, 48,1 ; Caton le Jeune, $32,3$.

60. Les remarques de Klaus Tausend (Graz) m'ont permis d'élargir mes conceptions. Je souhaite le remercier ici de sa contribution à la discussion.

61. Les deux pôles des écarts de conduite possibles pour un consul, la déviance de type Postumius et la déviance de type Sextius en tant que tribun consulaire, sont cités par Tite-Live dans le récit de l'éloge que reçut le consul T. Quinctius Capitolinus en 446, après que ce dernier, par son discours, fut parvenu à convaincre le peuple de s'enrôler. Afin de valoriser davantage la performance de Quinctius, on insiste en négatif, de manière très intéressante, sur l'attitude des autres consuls (Tite-Live, 3, 69, 4) : Alios consules aut per proditionem dignitatis patrum plebi adulatos aut acerbe tuendo iura ordinis asperiorem donando multitudinem fecisse.

62. Toute l'institution du senatus consultum ultimum se fonde sur l'hypothèse que les consuls se rangeront à l'orientation du Sénat. Ce principe fonctionna même en 100, lorsque le consul Marius, qui avait longtemps coopéré avec le tribun Appuleius Saturninus, fit appliquer le senatus consultum ultimum contre son ancien partenaire.

63. Suétone, Auguste, 99, 1: Supremo die [...] et admissos amicos percontatus, ecquid iis videretur mimum vitae commode transegisse, adiecit et clausulam :

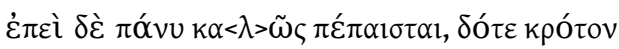

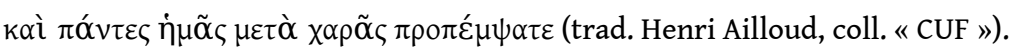

Voir Dion Cassius, 56, 30, 4. L'expression me paraît restituer très clairement la réalité des manières de l'empereur, si bien que son sens s'étend bien au-delà d'un mouvement de douce sérénité face à la futilité des actions humaines, auquel on l'assimile généralement (voir par exemple Crusius [1914], p. 320 ; Gugel [1977], p. 97). Voir aussi Bleicken (1998), p. 663. 
INDEX

Mots-clés : République romaine, institutions

Schlüsselwörter : römische Republik, Institutionen

\section{AUTEURS}

MARTIN JEHNE

Martin Jehne est professeur d'histoire ancienne à l'Université Technique de Dresde. Pour plus d'informations, voir la notice suivante. 